\title{
Periodic flats and group actions on locally symmetric spaces
}

\author{
GRIGORI AVRAMIDI
}

\begin{abstract}
We use maximal periodic flats to show that on a finite volume irreducible locally symmetric manifold of dimension $\geq 3$, no metric has more symmetry than the locally symmetric metric. We also show that if a finite volume metric is not locally symmetric, then its lift to the universal cover has discrete isometry group.
\end{abstract}

$57 \mathrm{~S} 15,57 \mathrm{~S} 20$

\section{Introduction}

The goal of this paper is to give some new results of the flavor that 'the locally symmetric metric on a locally symmetric manifold is the most symmetric metric on that manifold'. Earlier work, reviewed below, establishes such results for compact manifolds. Our goal here is to dispense with compactness, resulting in new obstacles and phenomena. Let $(M, g)$ be a complete Riemannian manifold homeomorphic to a finite volume, nonpositively curved locally symmetric space with no local torus factors. In this paper we prove that the metric $g$ has no homotopically trivial isometries. More precisely, the isometry group Isom $(M, g)$ acts on free homotopy classes of loops and this gives an action homomorphism

$$
\rho: \operatorname{Isom}(M, g) \rightarrow \operatorname{Out}\left(\pi_{1} M\right)
$$

Theorem 1 The homomorphism $\rho$ is injective.

If, in addition, the locally symmetric space is irreducible and of dimension $\geq 3$, then Margulis-Mostow-Prasad rigidity shows that the group $\operatorname{Out}\left(\pi_{1} M\right)$ is represented by isometries of the locally symmetric metric $h_{\text {sym }}$. Thus, $\operatorname{Isom}(M, g)<\operatorname{Isom}\left(M, h_{\text {sym }}\right)$ so that, in a sense, the locally symmetric metric has the most symmetry. If the metric $g$ has finite volume, then we also get the following dichotomy for the isometry group of the universal cover.

Theorem 2 Let $\left(M, h_{\text {sym }}\right)$ be a finite volume, irreducible, locally symmetric manifold of dimension $\geq 3$. Suppose that $M$ has no local torus factors. If $g$ is any complete, finite volume Riemannian metric on $M$, then either: 
- $g$ is a constant multiple of the locally symmetric metric, or

- the isometry group of the universal cover $\operatorname{Isom}(\widetilde{M}, \widetilde{g})$ is discrete and contains $\pi_{1} M$ as a subgroup of index $\leq \operatorname{vol}\left(M, h_{\mathrm{sym}}\right) / \varepsilon\left(h_{\mathrm{sym}}\right)$.

The constant $\varepsilon\left(h_{\mathrm{sym}}\right)$ is the volume of the smallest locally symmetric orbifold covered by $\left(M, h_{\text {sym }}\right)$. It does not depend on the metric $g$. The assumption that $g$ has finite volume turns out to be necessary. In fact, one can construct a complete infinite volume metric on $M$ such that the isometry group of the universal cover contains $\pi_{1} M * \mathbb{Z}$ as a discrete subgroup (see section 12 of Avramidi [1]).

\section{Related work}

For closed locally symmetric spaces, Theorem 1 was proved by Borel (published by Conner and Raymond, Theorem 3.2 of [5]) and Theorem 2 was proved by Farb and Weinberger (Theorem 1.7 of [7]). For finite volume locally symmetric spaces of non-zero Euler characteristic, Theorem 2 is proved (using $L^{2}$ cohomology rather than maximal periodic flats) in [1]. For noncompact locally symmetric spaces with vanishing Euler characteristic, both theorems are new.

One also has results similar to Theorem 2 for aspherical manifolds (or orbifolds) which are not locally symmetric. The analogous result for moduli spaces of algebraic curvesRoyden's Theorem - is treated in [1] and Farb and Weinberger [8]. For a class of closed aspherical manifolds which are tiled by locally symmetric spaces (piecewise locally symmetric spaces) Theorem 2 is proved by Tam Nguyen Phan in [14]. For more general aspherical Riemannian and Lorentz manifolds, one has results about the structure of isometry groups ([7] and Melnick [11]), but no quantitative bound (depending only on the topology of $M$ ) on how many isometries of the universal cover are not covering translations, ie, on the size of $\operatorname{Isom}(\widetilde{M}, \widetilde{g}) / \pi_{1} M$ (see Conjecture 1.6 in [7]).

\section{Acknowledgements}

I would like to thank my advisor Shmuel Weinberger for kindling my interest in isometries of aspherical manifolds, and for his guidance. I would also like to thank Benson Farb for a number of inspiring conversations, and Tam Nguyen Phan for drawing my attention to the paper Pettet and Souto [15].

\section{Maximal periodic flats in locally symmetric spaces}

Our proof of Theorem 1 is based on a result of Pettet and Souto [15], which says that the fundamental group of a locally symmetric space contains a free abelian subgroup that 'moves the boundary as much as possible'. We recall it now. 
Suppose that $G$ is a connected semisimple Lie group with no compact or Euclidean factors. Let $K<G$ be a maximal compact and $\Gamma<G$ a torsion free lattice. Suppose that $r:=\operatorname{rank}_{\mathbb{R}} G$ is the real rank of $G$. By a maximal $\Gamma$-periodic flat we will mean a totally geodesic $r$-dimensional flat subspace $\mathbb{R}^{r} \subset G / K$ that is invariant under some free abelian subgroup $\mathbb{Z}^{r}<\Gamma .1$

We will use the following facts. For an arithmetic locally symmetric space, these are standard (see Borel and Serre [3]). For general locally symmetric spaces, these facts are described in [15]. The unpublished manuscript Morris [12] is a general reference on arithmetic groups.

(1) The symmetric space $G / K$ has a maximal $\Gamma$-periodic flat.

(2) There is a number $q=\operatorname{rank}_{\mathbb{Q}}(M)$ called the $\mathbb{Q}$-rank of the locally symmetric space $M:=\Gamma \backslash G / K$ and a $(q-1)$-dimensional simplicial complex $\Delta_{\mathbb{Q}}(M)$ on which the group $\Gamma$ acts simplicially. The complex is called the rational Tits building of the locally symmetric space $M$. It is homotopy equivalent to an infinite wedge of $(q-1)$-spheres, ie, $\Delta_{\mathbb{Q}}(M) \cong \bigvee S^{q-1}$.

(3) The locally symmetric space $M$ is the interior of a compact manifold $\bar{M}$ with boundary $\partial \vec{M}$. There is a $\Gamma$-equivariant homotopy equivalence:

$$
\partial \widetilde{\bar{M}} \rightarrow \Delta_{\mathbb{Q}}(M)
$$

For the rest of this paper, we will use $\mathbb{R}^{r}$ to denote a fixed maximal $\Gamma$-periodic flat and $\mathbb{Z}^{r}<\Gamma$ a free abelian subgroup of rank $r$ which leaves that flat invariant. Pettet and Souto prove the following:

Theorem 3 (Proposition 5.5 in [15]) The stabilizer $\operatorname{Stab}_{\mathbb{Z}^{r}}\left(\sigma_{k}\right)$ of a $k$-simplex in the building $\Delta_{\mathbb{Q}}(M)$ is a free abelian group of rank $\leq r-k-1$.

Remark 1 Heuristically, the stabilizer spans a torus, which is complementary to the $(k+1)$-dimensional $\mathbb{Q}$-split torus corresponding to $\sigma_{k}$, and together they span a torus of $\operatorname{rank}^{\operatorname{rank}_{\mathbb{Z}}}\left(\operatorname{Stab}_{\mathbb{Z}^{r}}\left(\sigma_{k}\right)\right)+k+1 \leq r$.

Using this and a theorem of McMullen (6.1 in [15]), Pettet and Souto show that a maximal periodic flat cannot be homotoped to the boundary. On the level of universal covers, their theorem is:

\footnotetext{
${ }^{1}$ The image of $\mathbb{R}^{r}$ under $G / K \rightarrow \Gamma \backslash G / K$ is what [15] call a maximal periodic flat.
} 
Theorem 4 (Theorem 1.2 in [15]) With the notation above, let

$$
\mathbb{R}^{r} \subset G / K=\widetilde{M} \subset \widetilde{\bar{M}}
$$

be a maximal $\Gamma$-periodic flat invariant under a free abelian subgroup $\mathbb{Z}^{r}<\Gamma$. Then, $\mathbb{R}^{r}$ cannot be $\mathbb{Z}^{r}$-equivariantly homotoped into the boundary $\partial \widetilde{\bar{M}}$.

The following consequence, pointed out to us by Tam Nguyen Phan, is the reason for our initial interest in maximal periodic flats.

Corollary 5 If $(M, g)$ is a complete, Riemannian manifold homeomorphic to a finite volume, aspherical locally symmetric space with no local torus factors, then the group of homotopically trivial isometries $K:=\operatorname{ker}\left(\rho: \operatorname{Isom}(M, g) \rightarrow \operatorname{Out}\left(\pi_{1} M\right)\right)$ is a compact Lie group.

Proof The group $K$ is a Lie group by the Myers-Steenrod Theorem [13]. Let $N \subset \bar{M}$ be a collar neighborhood of the boundary. If the group $K$ is not compact, then there is a homotopically trivial isometry $\phi$ that sends $\bar{M} \backslash N$ into the collar neighborhood of the boundary $N$. This isometry defines a $\mathbb{Z}^{r}$-equivariant homotopy of a maximal periodic flat into a neighborhood of the boundary, contradicting Theorem 4.

We will deduce Theorem 4 from Theorem 3 using equivariant homology instead of McMullen's Theorem. This seems to be a simplification, and in any case we need it for our proof of Theorem 1 .

First, we will restate Theorem 4 in a slightly different form. Notice that if the flat $\mathbb{R}^{r}$ can be $\mathbb{Z}^{r}$-equivariantly homotoped to the boundary, then we have a $\mathbb{Z}^{r}$-equivariant map:

$$
s: \mathbb{R}^{r} \rightarrow \partial \widetilde{\bar{M}} \rightarrow \Delta_{\mathbb{Q}}(M)
$$

Give the product $\mathbb{R}^{r} \times \Delta_{\mathbb{Q}}(M)$ the diagonal $\mathbb{Z}^{r}$-action. By projecting onto the first factor, we get a bundle $\left(\mathbb{R}^{r} \times \Delta_{\mathbb{Q}}(M)\right) / \mathbb{Z}^{r} \rightarrow \mathbb{T}^{r}$ over the torus $\mathbb{T}^{r}$ with fibre $\Delta_{\mathbb{Q}}(M)$. The $\mathbb{Z}^{r}$-equivariant map

$$
\mathbb{R}^{r} \stackrel{v \mapsto(v, v)}{\longrightarrow} \mathbb{R}^{r} \times \mathbb{R}^{r} \stackrel{i d \times s}{\longrightarrow} \mathbb{R}^{r} \times \Delta_{\mathbb{Q}}(M)
$$

gives a section of this bundle. Thus, Theorem 4 follows from:

Theorem 6 Let $\mathbb{Z}^{r}<\Gamma$ be a free abelian subgroup leaving invariant a maximal $\Gamma$-periodic flat $\mathbb{R}^{r} \subset \widetilde{M}$. Then, the bundle

$$
\Delta_{\mathbb{Q}}(M) \rightarrow \frac{\mathbb{R}^{r} \times \Delta_{\mathbb{Q}}(M)}{\mathbb{Z}^{r}} \rightarrow \mathbb{T}^{r}
$$

does not have a section. 
Proof We will show that $\left(\mathbb{R}^{r} \times \Delta_{\mathbb{Q}}(M)\right) / \mathbb{Z}^{r}$ has no homology in dimension $r$, which implies that (2) cannot have a section. (If there is a section $s$, then $s\left(\mathbb{T}^{r}\right)$ represents a non-trivial homology class.) Let $C_{*}\left(\mathbb{R}^{s}\right)$ and $C_{*}\left(\Delta_{\mathbb{Q}}(M)\right)$ be the simplicial chain complexes of $\mathbb{Z}^{r}$-equivariant triangulations on $\mathbb{R}^{r}$ and $\Delta_{\mathbb{Q}}(M)$, respectively. The double complex

$$
E_{s, t}^{0}:=C_{S}\left(\mathbb{R}^{r}\right) \otimes_{\mathbb{Z}^{r}} C_{t}\left(\Delta_{\mathbb{Q}}(M)\right)
$$

has the spectral sequence

$$
E_{s, t}^{1}:=H_{S}\left(\mathbb{Z}^{r} ; C_{t}\left(\Delta_{\mathbb{Q}}(M)\right)\right) \Longrightarrow H_{s+t}\left(\frac{\mathbb{R}^{r} \times \Delta_{\mathbb{Q}}(M)}{\mathbb{Z}^{r}}\right)
$$

associated to it. We will use the fact that $\mathbb{Z}^{r}$ acts with small stabilizers on the rational Tits building $\Delta_{\mathbb{Q}}(M)$. Note that

$$
\begin{aligned}
E_{s, t}^{1}: & =H_{S}\left(\mathbb{Z}^{r} ; C_{t}\left(\Delta_{\mathbb{Q}}(M)\right)\right) \\
& =H_{s}\left(\mathbb{Z}^{r} ; \bigoplus_{\sigma_{t}} \mathbb{Z}^{r} \cdot \sigma_{t}\right) \\
& =\bigoplus_{\sigma_{t}} H_{s}\left(\mathbb{Z}^{r} ; \mathbb{Z}^{r} \cdot \sigma_{t}\right) \\
& =\bigoplus_{\sigma_{t}} H_{s}\left(\operatorname{Stab}_{\mathbb{Z}^{r}}\left(\sigma_{t}\right)\right)
\end{aligned}
$$

where the sum is taken over all $\mathbb{Z}^{r}$-orbits of $t$-simplices. By the result of Pettet and Souto (Theorem 3), $\operatorname{Stab}_{\mathbb{Z}^{r}}\left(\sigma_{t}\right)$ is a free abelian group of rank $\leq r-t-1$, so we find that $E_{s, t}^{1}=0$ for $s \geq r-t$, ie, for $s+t \geq r$. Since this spectral sequence converges to the homology of $\left(\mathbb{R}^{r} \times \Delta_{\mathbb{Q}}(M)\right) / \mathbb{Z}^{r}$, we find that

$$
H_{k}\left(\frac{\mathbb{R}^{r} \times \Delta_{\mathbb{Q}}(M)}{\mathbb{Z}^{r}}\right)=0 \text { for } k \geq r .
$$

This proves Theorem 6 .

\section{Homology of $\partial \tilde{\bar{M}} / \mathbb{Z}^{r}$}

For future use, we rephrase the above computation in terms of the homology of the Borel-Serre boundary. We have homotopy equivalences

$$
\partial \widetilde{\bar{M}} / \mathbb{Z}^{r} \leftarrow\left(\mathbb{R}^{r} \times \partial \widetilde{\bar{M}}\right) / \mathbb{Z}^{r} \rightarrow\left(\mathbb{R}^{r} \times \Delta_{\mathbb{Q}}(M)\right) / \mathbb{Z}^{r} .
$$

The left map is a homotopy equivalence because it is the projection map of a bundle with fibre $\mathbb{R}^{r}$. The right map is the obvious homotopy equivalence obtained from the 
$\mathbb{Z}^{r}$-equivariant homotopy equivalence $\partial \widetilde{\bar{M}} \rightarrow \Delta_{\mathbb{Q}}(M)$. Thus, equation (5) shows that the homology of the Borel-Serre boundary of the $\mathbb{Z}^{r}$ cover vanishes in dimensions $\geq r$, ie:

$$
H_{k}\left(\partial \widetilde{\bar{M}} / \mathbb{Z}^{r}\right)=0 \text { for } k \geq r
$$

This equation, the long exact homology sequence

$$
\cdots \rightarrow H_{*}\left(\widetilde{\bar{M}} / \mathbb{Z}^{r}\right) \rightarrow H_{*}\left(\widetilde{\bar{M}} / \mathbb{Z}^{r}, \partial \widetilde{\bar{M}} / \mathbb{Z}^{r}\right) \rightarrow H_{*-1}\left(\partial \widetilde{\bar{M}} / \mathbb{Z}^{r}\right) \rightarrow \cdots
$$

and the fact that $\widetilde{\bar{M}} / \mathbb{Z}^{r}$ has no homology above dimension $r$ (it is homotopy equivalent to the $r$-torus) implies that:

$$
H_{k}\left(\widetilde{\bar{M}} / \mathbb{Z}^{r}, \partial \widetilde{\bar{M}} / \mathbb{Z}^{r}\right)=0 \text { for } k>r
$$

Everything we have said so far is valid for homology with coefficients $\kappa$ in $\mathbb{Z}, \mathbb{R}$ and also with coefficients in the field $\mathbb{F}_{p}$ of $p$ elements. It is useful to rewrite this in terms of homology with local coefficients in the $[\Gamma]$ module $\left[\Gamma / \mathbb{Z}^{r}\right]$ :

$$
\begin{aligned}
H_{k}\left(\partial \bar{M} ;\left[\Gamma / \mathbb{Z}^{r}\right]\right)=0 & \text { for } k \geq r \\
H_{k}\left(\bar{M}, \partial \bar{M} ;\left[\Gamma / \mathbb{Z}^{r}\right]\right)=0 & \text { for } k>r
\end{aligned}
$$

\section{Homotopically trivial $\mathbb{Z} / p$-actions}

\subsection{Outline of proof of Theorem 1}

We have seen that the group $K=\operatorname{ker}(\rho)$ of homotopically trivial isometries is a compact Lie group (Corollary 5). Thus, to show this group is trivial we only need to check that there are no elements of prime order $p$. In other words, we need to show that the locally symmetric space $M$ has no non-trivial, homotopically trivial $\mathbb{Z} / p$-actions. We lift the $\mathbb{Z} / p$-action to the cover $\widetilde{\bar{M}} / \mathbb{Z}^{r}$, look at the fixed point set 'near the boundary' of this cover, and use (10) and (11) (with $\kappa=\mathbb{F}_{p}$ ) to show the fixed point set is everything.

The $\mathbb{Z} / p$-action may not extend to the Borel-Serre boundary of $M$, so we need to replace homology of the boundary (10) and homology relative to the boundary (11) by homology of the end and homology with closed supports, respectively. We recall these notions in the next two subsections. 


\subsection{Homology with closed supports}

Given a $\Gamma$-cover $\tilde{X} \rightarrow X$, denote by $C_{*}^{\text {cl }}(X ; V)$ the complex of chains with closed support on $X$ and coefficients in the $[\Gamma]$-module $V$. More precisely, first define $C_{*}^{\mathrm{cl}}(X ;[\Gamma])$ as the complex of those chains on the cover $\tilde{X}$ that for every compact set $K \subset \widetilde{X}$ meet only finitely many $\Gamma$-translates of $K$. Then, define $C_{*}^{\text {cl }}(X ; V):=$ $C_{*}^{\mathrm{cl}}(X ;[\Gamma]) \otimes_{[\Gamma]} V$.

If $M$ is the interior of a compact manifold with boundary, $\partial M \times(0, \infty)$ is an open neighborhood of the boundary, and $M_{0}:=M \backslash(\partial M \times(0, \infty))$ its complement, then the relative homology of the pair $\left(M_{0}, \partial M_{0}\right)$ is isomorphic to homology with closed supports on $M$ via:

$$
\begin{aligned}
H_{*}\left(M_{0}, \partial M_{0} ; V\right) & \cong H_{*}^{\mathrm{cl}}(M ; V) \\
(c, \partial c) & \mapsto c \cup_{\partial c} \partial c \times[0, \infty)
\end{aligned}
$$

One way to see this is to note that the map is Poincare dual (V.9.2 in [4]) to the cohomology isomorphism $H^{m-*}(M ; \mathcal{O} \otimes V) \cong H^{m-*}\left(M_{0} ; \mathcal{O} \otimes V\right)$, where $\mathcal{O}$ is the orientation module.

\subsection{Homology of the end}

The complex $C_{*}^{e}(X ; V)$ of chains on the end of $X$ is defined to be the quotient:

$$
0 \rightarrow C_{*+1}(X ; V) \rightarrow C_{*+1}^{\mathrm{cl}}(X ; V) \rightarrow C_{*}^{e}(X ; V) \rightarrow 0
$$

Denote the homology of this complex by $H_{*}^{e}(X ; V):=H_{*}\left(C_{*}^{e}(X ; V)\right)$. Associated to the short exact sequence of chain complexes (14) there is a long exact homology sequence:

$$
\cdots \rightarrow H_{*+1}^{\mathrm{cl}}(X ; V) \rightarrow H_{*}^{e}(X ; V) \rightarrow H_{*}(X ; V) \rightarrow \cdots
$$

Putting together the long exact homology sequence of the pair $\left(M_{0}, \partial M_{0}\right)$ with the long exact homology sequence (15), we get a commutative diagram:

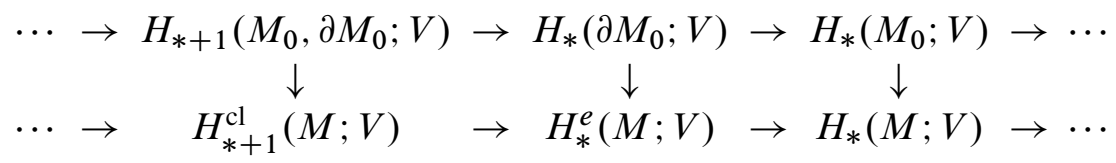

The left vertical arrow is an isomorphism by (12) and the right vertical arrow is an isomorphism because (ordinary) homology is homotopy invariant, so the middle arrow is an isomorphism:

$$
\begin{aligned}
H_{*}\left(\partial M_{0} ; V\right) & \cong H_{*}^{e}(M ; V) \\
a & \mapsto a \times[0, \infty)
\end{aligned}
$$


Putting together (10), (11), (12) and (16) we get:

$$
\begin{array}{ll}
H_{k}^{e}\left(M ;\left[\Gamma / \mathbb{Z}^{r}\right]\right)=0 & \text { for } k \geq r \\
H_{k}^{\mathrm{cl}}\left(M ;\left[\Gamma / \mathbb{Z}^{r}\right]\right)=0 & \text { for } k>r
\end{array}
$$

\subsection{Smith theory}

In this subsection we recall the Smith theory we need to compare the homology of the fixed set of a homotopically trivial $\mathbb{Z} / p$ (or $S^{1}$ )-action to the homology of the ambient space. One has the following amplification of the classical Smith theorem, which extends results of Conner and Raymond (see the appendix of [5]).

Theorem 7 (see Theorem 9 in [1]) Let $M$ be an $m$-dimensional smooth aspherical manifold and $\widetilde{M}$ its universal cover. Suppose we have a smooth $(\mathbb{Z} / p)^{n}$-action on $\widetilde{M}$ that commutes with the covering action of the fundamental group $\Gamma:=\pi_{1} M$. Let $F \subset M$ be the fixed point set of the projected $(\mathbb{Z} / p)^{n}$-action on $M$. Let $V$ be an $\mathbb{F}_{p}[\Gamma]-$ module. Then, the inclusion of the fixed point set induces isomorphisms on homology and cohomology with coefficients in $V$, ie:

$$
\begin{aligned}
H_{*}(F ; V) & \cong H_{*}(M ; V) \\
H^{*}(M ; V) & \cong H^{*}(F ; V)
\end{aligned}
$$

If we have a smooth $\left(S^{1}\right)^{n}$-action on $\widetilde{M}$ that commutes with $\Gamma$ then the same isomorphisms are true with coefficients in any $\mathbb{R}[\Gamma]$-module $V$.

Proof For $\mathbb{Z} / p$-actions this is Theorem 9 in [1]. For $(\mathbb{Z} / p)^{n}$-actions $\left(\left(S^{1}\right)^{n}\right.$-actions $)$ this is proved in exactly the same way, starting with the fact that the fixed set of a $(\mathbb{Z} / p)^{n}$-action $\left(\left(S^{1}\right)^{n}\right.$-action) on the contractible manifold $\widetilde{M}$ is $\mathbb{F}_{p}$-acyclic $(\mathbb{R}$ acyclic).

The cohomology isomorphism can be translated to an isomorphism for homology with closed supports.

Corollary 8 If the fixed point set has dimension $f$, then we have an isomorphism of homology with closed supports:

$$
H_{*+m-f}^{\mathrm{cl}}(M ; V) \cong H_{*}^{\mathrm{cl}}(F ; V)
$$

Remark 2 Geometrically, the isomorphism (21) is obtained by sending a cycle $c$ on $M$ to the transverse intersection $c \cap F$ on $F$. 
Proof Denote by $\mathcal{O}_{M}$ and $\mathcal{O}_{F}$ the orientation [ $\left.\Gamma\right]$-modules on $M$ and $F$, respectively. If $\kappa=\mathbb{F}_{2}$, then these modules are trivial, so they are equal. Else, then the normal bundle of the fixed point set (to an $\left(S^{1}\right)^{n}$-action or $(\mathbb{Z} / p)^{n}$-action for an odd prime $p$ ) is orientable, so again the orientation modules are equal. Combining this with the Poincaré duality isomorphisms (Theorem V.9.2 of [4]),

$$
\begin{aligned}
H_{*}^{\mathrm{cl}}(M ; V) & \cong H^{m-*}\left(M ; \mathcal{O}_{M} \otimes V\right) \\
H_{*}^{\mathrm{cl}}(F ; V) & \cong H^{f-*}\left(F ; \mathcal{O}_{F} \otimes V\right)
\end{aligned}
$$

and the cohomology isomorphism (20) proves (21).

\subsection{Proof of Theorem 1}

Since $M$ is a locally symmetric space with no local torus factors, its fundamental group is centerless (10.3.10 of Eberlein [6]). Thus, by Proposition 9, the homotopically trivial $\mathbb{Z} / p$-action on $M$ lifts to a $\mathbb{Z} / p$-action on $\widetilde{M}$ that commutes with the covering action of the fundamental group $\Gamma$. This lets one apply the Smith theory from the previous subsection.

Let $F \subset M$ be the fixed set of $\mathbb{Z} / p$ acting on $M$. Denote by $f$ and $m$ the dimensions of $F$ and $M$, respectively. Our goal is to show that $f=m$, ie, that the fixed point set has the same dimension as the manifold $M$. Since the fixed point set is a closed submanifold of $M$, and $M$ is connected, this will show that it is everything, ie, that the $\mathbb{Z} / p$-action is trivial.

In this proof we will use $\kappa=\mathbb{F}_{p}$ coefficients. The inclusion $F \hookrightarrow M$ induces maps on homology, homology with closed supports and homology of the end with coefficients in $V:=\mathbb{F}_{p}\left[\Gamma / \mathbb{Z}^{r}\right]$. These fit together in the commutative diagram

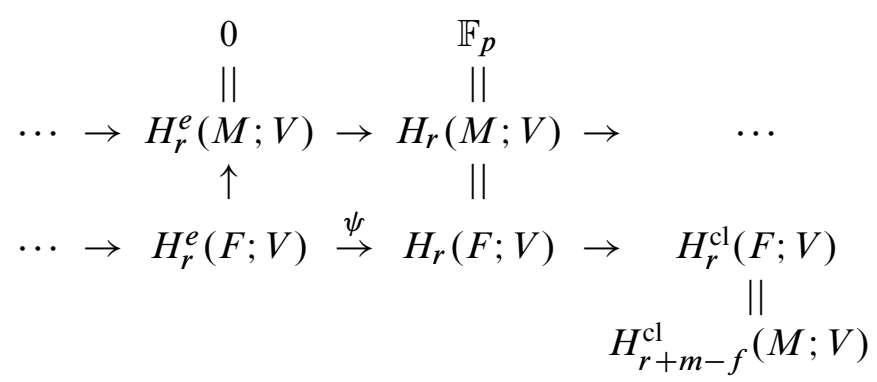

whose rows are long exact homology sequences. The top row is computed using (17) and the fact that $H_{r}\left(M ; \mathbb{F}_{p}\left[\Gamma / \mathbb{Z}^{r}\right]\right)=H_{r}\left(\widetilde{M} / \mathbb{Z}^{r} ; \mathbb{F}_{p}\right)=\mathbb{F}_{p}$. The vertical isomorphisms are the Smith theory isomorphisms (19) and (21). If $m>f$ then equation (18) shows that $\psi$ is onto. This is a contradiction, since the commutative diagram shows that $\psi$ is 
the zero map. Thus, $m=f$, which means the fixed point set is the entire manifold $M$, ie, the homotopically trivial $\mathbb{Z} / p$-action is actually trivial. This completes the proof of Theorem 1.

\section{Proof of Theorem 2}

The following proof uses methods developed in [7]. It closely follows the argument given in sections 7,8 and 10 of [1] and is collected here for the convenience of the reader. The main new ingredient is Theorem 1 . We will repeatedly use the following standard fact about group extensions.

Proposition 9 (IV.8.8. in Mac Lane [10]) A group extension

$$
1 \rightarrow A<B \stackrel{\pi}{\rightarrow} C \rightarrow 1
$$

is determined by:

(1) the conjugation representation $\rho: C \rightarrow \operatorname{Out}(A)$, and

(2) a cohomology class in the group $H^{2}(C ; Z(A))$.

In particular, if both the center $Z(A)$ and the representation $\rho$ are trivial, then the extension splits as a product. In this case:

- there is a projection $B \stackrel{\varphi}{\rightarrow} A$ which restricts to the identity map on $A$, and

- a section $C \stackrel{s}{\rightarrow} B$ whose image $s(C)$ commutes with $A$.

Let $\Gamma:=\pi_{1} M$ be the fundamental group, $I:=\operatorname{Isom}(\widetilde{M}, \widetilde{g})$ the isometry group of the universal cover and $I_{0}$ its connected component. The Myers-Steenrod theorem [13] implies $I$ is a Lie group acting smoothly and properly on $\widetilde{M}$. Let $K<I$ be a compact Lie group of isometries.

Step 1 If $\boldsymbol{\Gamma}$ commutes with the compact Lie group $\boldsymbol{K}$, then $\boldsymbol{K}=\mathbf{1}$

To show that $K$ is trivial, it suffices to show that it has no elements $\phi$ of prime order $p$. Since $\phi$ commutes with $\Gamma$, it descends to a homotopically trivial $\mathbb{Z} / p$-action on the locally symmetric space $M$. We showed in the proof of Theorem 1 that there are no such actions. 


\section{Step 2 If $\boldsymbol{\Gamma}$ normalizes the compact Lie group $\boldsymbol{K}$, then $\boldsymbol{K}=\mathbf{1}$}

We will successively eliminate characteristic compact subgroups. Denote by $K_{0}$ the identity component of the compact Lie group $K$. It can be expressed as an extension

$$
1 \rightarrow K_{0}^{\mathrm{sol}} \rightarrow K_{0} \rightarrow K_{0}^{\mathrm{ss}} \rightarrow 1
$$

of the maximal normal connected solvable subgroup $K_{0}^{\text {sol }}<K$ by a semisimple group $K_{0}^{\text {ss }}$. The group $\Gamma$ acts by conjugation on $K$, hence also on the connected component $K_{0}$, and on its characteristic subgroup $K_{0}^{\text {sol }}$. The group $K_{0}^{\text {sol }}$ is compact, connected and solvable, so it is a torus $\left(S^{1}\right)^{n}$. It contains a finite subgroup $(\mathbb{Z} / p)^{n}<\left(S^{1}\right)^{n}$ consisting of the elements of order 1 and $p$. This subgroup is characteristic, so $\Gamma$ acts on it. Since $(\mathbb{Z} / p)^{n}$ is finite, a finite index subgroup $\Gamma^{\prime}<\Gamma$ acts trivially on it. In other words, $\Gamma^{\prime}$ commutes with $(\mathbb{Z} / p)^{n}$. Now Step 1 implies that $n=0$ so that $K_{0}$ is semisimple. Notice that:

- The center $Z\left(K_{0}\right)$ is trivial: Indeed, the center is finite since the group $K_{0}$ is semisimple. Thus, a finite index subgroup of $\Gamma$ commutes with it. Hence, Step 1 implies the center is trivial.

- Conjugation gives a homomorphism $\rho: \Gamma \rightarrow \operatorname{Out}\left(K_{0}\right)$ from $\Gamma$ to the group $\operatorname{Out}\left(K_{0}\right)$ of topological outer automorphisms of $K_{0}$. This group is finite because $K_{0}$ is semisimple with trivial center. Thus, replacing $\Gamma$ with a finite index subgroup if necessary, we may assume the homomorphism $\rho$ is trivial.

Now, Proposition 9 implies that the extension

$$
1 \rightarrow K_{0} \rightarrow K_{0} \Gamma \rightarrow \Gamma \rightarrow 1
$$

has a projection $\varphi: K_{0} \Gamma \rightarrow K_{0}$ and section $s: \Gamma \rightarrow K_{0} \Gamma$ whose image commutes with $K_{0}$. Note that the section $s(\Gamma)$ is discrete because it is a compact perturbation of $\Gamma$.

If $r \leq 1$ then eliminating any $S^{1}$-action (and hence $K_{0}$-action) commuting with $s(\Gamma)$ is elementary: in this case the homological dimension of $\Gamma$ is $\geq m-1$ so Smith theory implies the fixed set has dimension $\geq m-1$. Since the fixed set of $S^{1}$ has even codimension, the action must be trivial. Now, we deal with the $r \geq 2$ case.

Notation Denote the image of the section s by $\Gamma_{*}$, let $\mathbb{Z}_{*}^{r}:=s\left(\mathbb{Z}^{r}\right)$ and let $M_{*}:=$ $\widetilde{M} / \Gamma_{*}$. The closure of the image of $\varphi: \mathbb{Z}^{r} \rightarrow K_{0}$ is an abelian subgroup and, passing to a finite index subgroup of $\mathbb{Z}^{r}$ if necessary, we may assume that the closure is a torus $\overline{\varphi\left(\mathbb{Z}^{r}\right)}=\left(S^{1}\right)^{n}=: T$. Let $F_{*} \subset M_{*}$ be the fixed point set of $T$. Let $V:=\mathbb{R}\left[\Gamma / \mathbb{Z}^{r}\right]$. Pick a homotopy equivalence $h$ between $M$ and $M^{*}$ that on the universal cover $\widetilde{M}$ 
takes the $\Gamma$-action to the $\Gamma_{*}$-action. ${ }^{2}$ This gives an identification $V \cong \mathbb{R}\left[\Gamma_{*} / \mathbb{Z}_{*}^{r}\right]$. The manifolds $M$ and $M_{*}$ have a common (orbifold) quotient $M_{*} / K_{0}=\widetilde{M} / K_{0} \Gamma$. Let $E_{t}$ be an exhaustion of the end of $M_{*} / K_{0}, \widetilde{E}_{t}$ its lift to the universal cover, $M_{t}$ and $M_{* t}$ its lifts to $M$ and $M_{*}$, respectively, and $F_{* t}:=M_{* t} \cap F_{*}$ its restriction to the fixed point set.

Remark 3 Although $M$ and $M_{*}$ are homotopy equivalent, it is not at all clear that they are properly homotopy equivalent, so we cannot directly apply Step 1 to $M_{*}$ to eliminate $K_{0}$.

Assume that the torus $T$ is non-trivial, so that its fixed set has dimension $f<m$. Starting from this, we will arrive at a contradiction. The key point is that the actions of $\mathbb{Z}^{r}$ and $\mathbb{Z}_{*}^{r}$ differ by elements of $T$, so on the fixed point set $\widetilde{F}_{*} \subset \widetilde{M}$ of $T$ the two actions are identical. Thus, we get a map $\widetilde{F}_{*} / \mathbb{Z}_{*}^{r} \rightarrow \widetilde{M} / \mathbb{Z}^{r}$, which is an $\mathbb{R}$-homology isomorphism (the proof of Theorem 9 in [1] applies because $\widetilde{F}_{*}$ is $\mathbb{R}$-acyclic). Pick a large enough $t$ so that $M_{t}$ is contained in a collar neighborhood of the boundary $\partial \bar{M}$. Then the map from the fixed point set restricts to a map:

$$
\widetilde{F_{* t}} / \mathbb{Z}_{*}^{r} \rightarrow \widetilde{M_{t}} / \mathbb{Z}^{r} \rightarrow \partial \widetilde{\bar{M}} / \mathbb{Z}^{r}
$$

Putting these observations together, we get the commutative diagram

$$
\begin{gathered}
0=H_{r}\left(\partial \widetilde{\bar{M}} / \mathbb{Z}^{r} ; \mathbb{R}\right) \longrightarrow H_{r}\left(\widetilde{M} / \mathbb{Z}^{r} ; \mathbb{R}\right)=\mathbb{R} \\
\uparrow \\
H_{r}\left(\widetilde{F}_{* t} / \mathbb{Z}_{*}^{r} ; \mathbb{R}\right) \longrightarrow H_{r}\left(\widetilde{F}_{*} / \mathbb{Z}_{*}^{r} ; \mathbb{R}\right)
\end{gathered}
$$

showing the bottom horizontal map is zero.

Claim The map $H_{r}^{e}\left(F_{*} ; V\right) \stackrel{\psi}{\rightarrow} H_{r}\left(F_{*} ; V\right)=\mathbb{R}$ is also zero

Proof The map $\psi$ is the connecting homomorphism arising from the short exact sequence of chain complexes (14). From the chain level definition, one sees that this homomorphism factors through

$$
H_{r}\left(\widetilde{F}_{* t} / \mathbb{Z}_{*}^{r} ; \mathbb{R}\right)=H_{r}\left(F_{* t} ; V\right) \rightarrow H_{*}\left(F_{*} ; V\right)=H_{r}\left(\widetilde{F}_{*} / \mathbb{Z}_{*}^{r} ; \mathbb{R}\right)
$$

for every $t$. Thus, it is the zero map.

${ }^{2}$ That is, $\widetilde{h}: \widetilde{M} \rightarrow \widetilde{M}$ with $\widetilde{h}(\gamma x)=s(\gamma) \tilde{h}(x)$. 
On the other hand, the long exact homology sequence (15) shows $\psi$ is onto since

$$
\begin{aligned}
H_{r}^{\mathrm{cl}}\left(F_{*} ; V\right) & \cong H^{f-r}\left(F_{*} ; \mathcal{O} \otimes V\right) & & \text { by Poincaré duality, } \\
& \cong H^{f-r}\left(M_{*} ; \mathcal{O} \otimes V\right) & & \text { by Smith theory with } \mathbb{R} \text {-coefficients }(20), \\
& \cong H^{f-r}(M ; \mathcal{O} \otimes V) & & \text { via the homotopy equivalence } h: M \rightarrow M_{*}, \\
& \cong H_{r+m-f}^{\mathrm{cl}}(M ; V) & & \text { by Poincaré duality, } \\
& =0 & & \text { by (18) since } f<m .
\end{aligned}
$$

This is a contradiction. Thus, the torus $T$ must be trivial, ie, $\mathbb{Z}^{r}$ is in the kernel of the homomorphism $\varphi$. Since $r \geq 2$ and $\Gamma$ is an irreducible lattice, the Margulis normal subgroup theorem (Theorem 8.1.2 in [19]) shows that the image of $\varphi$ is finite. Its kernel is a finite index subgroup $\Gamma^{\prime}<\Gamma$ that commutes with $K_{0}$. Now, we can apply Step 1 to $\Gamma^{\prime}$ to show that $K_{0}$ is trivial.

Remark 4 The argument actually shows that $\varphi(A)$ is finite for any subgroup $A<\Gamma$ that preserves some maximal $\Gamma$-periodic flat. One can probably deduce from this that $\varphi(\Gamma)$ is finite without invoking the normal subgroup theorem.

We have shown that $K$ is a finite group. Consequently, some finite index subgroup of $\Gamma$ commutes with it and yet another application of Step 1 shows $K$ is trivial. This completes the proof of Step 2.

Remark 5 We would like to mention two other ways to prove Step 2.

The first way is to observe that the commutative diagram (25) can be extended to a commutative diagram

$$
\begin{gathered}
H_{r}\left(\partial \widetilde{\bar{M}} / \mathbb{Z}^{r} ; \mathbb{R}\right) \longrightarrow H_{r}\left(\widetilde{M} / \mathbb{Z}^{r} ; \mathbb{R}\right) \\
\uparrow \\
H_{r}\left(\widetilde{E}_{t} / \mathbb{Z}_{*}^{r} ; \mathbb{R}\right) \longrightarrow H_{r}\left(\widetilde{M} / \mathbb{Z}_{*}^{r} ; \mathbb{R}\right)
\end{gathered}
$$

mapping from the homology of all of $\widetilde{M} / \mathbb{Z}_{*}^{r}$ (not just the fixed point set) with the vertical maps obtained by smearing chains out by the torus $T$ via Thurston's smearing method. ${ }^{3}$ From this diagram one can deduce that $H_{\geq r}^{e}\left(M_{*} ; \mathbb{R}\left[\Gamma_{*} / \mathbb{Z}_{*}^{r}\right]\right)=0$. Now one can do the argument in the proof of Theorem 1 with $(\kappa=\mathbb{R}$ coefficients) directly on the manifold $M_{*}$ to eliminate any homotopically trivial $S^{1}$-actions.

The second way is to do the action dimension + surgery argument from page 6 of [8] since one knows by Bestvina and Feighn [2] that lattices in locally symmetric spaces do not act properly discontinuously on contractible manifolds of smaller dimension.

\footnotetext{
${ }^{3}$ Smearing is described in section 6.2 of [18]. See also section 4.2 of [9].
} 


\section{Step $3 \boldsymbol{I}_{\mathbf{0}}$ is semisimple with trivial center and no compact factors}

The connected Lie group $I_{0}$ can be expressed as an extension

$$
1 \rightarrow I_{0}^{\mathrm{sol}} \rightarrow I_{0} \rightarrow I_{0}^{\mathrm{ss}} \rightarrow 1
$$

of the maximal connected solvable normal subgroup of $I_{0}$ by a semisimple group $I_{0}^{\text {ss }}$. The group $\Gamma_{0}:=\Gamma \cap I_{0}$ also has a unique maximal normal solvable subgroup [17], denoted $\Gamma_{0}^{\text {sol }}$.

Claim $\Gamma_{0}^{\text {sol }}$ is trivial The solvable group $\Gamma_{0}^{\text {sol }}$ is a characteristic subgroup of $\Gamma_{0}$, so it is a normal subgroup of $\Gamma$. On the other hand, recall that the fundamental group $\Gamma$ of a locally symmetric space with no local torus factors has no non-trivial abelian normal subgroups (10.3.10 of [6]), hence no non-trivial normal solvable subgroups. This proves the claim.

Farb and Weinberger [8] show that $\Gamma_{0}$ is a lattice in $I_{0}$. (While their arguments are stated for the mapping class group, their arguments only use the assumption that $\widetilde{g}$ has finite $\Gamma$-covolume and no particular properties of the mapping class group situation.) Then, they apply Prasad's Lemma 6 in [16] and get that the solvable part $I_{0}^{\text {sol }}$ is compact and the center of the semisimple part $Z\left(I_{0}^{\text {ss }}\right)$ is finite. Since the group $\Gamma$ acts on the solvable part $I_{0}^{\text {sol }}$, Step 2 implies the solvable part is trivial. In other words, $I_{0}=I_{0}^{\text {ss }}$ is semisimple. Since $\Gamma$ acts by conjugation on the center $Z\left(I_{0}^{\mathrm{ss}}\right)$ (a finite group), Step 2 implies that the center is trivial. Since the product of the compact factors is a compact characteristic subgroup of $I_{0}$, Step 2 implies that it is trivial. In summary, we have shown that $I_{0}$ is semisimple group with trivial center and no compact factors.

\section{Step 4}

The group generated by $I_{0}$ and $\Gamma$ is an extension:

$$
1 \rightarrow I_{0} \rightarrow\left\langle I_{0}, \Gamma\right\rangle \stackrel{\pi}{\rightarrow} \Gamma / \Gamma_{0} \rightarrow 1
$$

Since $I_{0}$ is semisimple with trivial center, its outer automorphism group is finite. Thus, the kernel of the conjugation homomorphism $\Gamma \rightarrow \operatorname{Out}\left(I_{0}\right)$ is a finite index subgroup $\Gamma^{\prime}<\Gamma$. Let $\Gamma_{0}^{\prime}=\Gamma^{\prime} \cap I_{0}$. Proposition 9 gives a projection $\varphi:\left\langle I_{0}, \Gamma^{\prime}\right\rangle \rightarrow I_{0}$, which restricts to the identity map on $I_{0}$. One easily checks that the product map

$$
\left\langle I_{0}, \Gamma^{\prime}\right\rangle \stackrel{\varphi \times \pi}{\longrightarrow} I_{0} \times \Gamma^{\prime} / \Gamma_{0}^{\prime}
$$

is an isomorphism that maps $\Gamma^{\prime}$ onto the product $\Gamma_{0}^{\prime} \times \Gamma^{\prime} / \Gamma_{0}^{\prime}$. Now, we use the hypothesis that $\Gamma^{\prime}$ is irreducible to conclude that either $\Gamma_{0}^{\prime}=1$ or $\Gamma_{0}^{\prime}$ is a finite index subgroup of $\Gamma^{\prime}$. We look at the two possibilities. 
Case $1 \Gamma_{0}^{\prime}<\Gamma^{\prime}$ is a finite index subgroup We will show that in this case $(M, g)$ is isometric to a locally symmetric space. Since $\Gamma_{0}$ is a lattice in the Lie group $I_{0}$, the finite index subgroup $\Gamma_{0}^{\prime}$ is also a lattice in $I_{0}$. Denote by $K<I_{0}$ a maximal compact subgroup. Then, the quotient $I_{0} / K$ is a symmetric space with no compact or Euclidean factors. Since $\Gamma_{0}^{\prime}$ is a finite index subgroup of $\Gamma$, Margulis-Mostow-Prasad rigidity implies the locally symmetric space $\Gamma_{0}^{\prime} \backslash I_{0} / K$ is isometric to a finite cover of $\left(M, h_{\text {sym }}\right)$. In particular, the two spaces have the same dimension. On the other hand, an orbit of the group $I_{0}$ acting on $\widetilde{M}$ has the form $I_{0} \cdot x=I_{0} / K_{x}$ for a compact subgroup $K_{x}<I_{0}$. We get

$$
\operatorname{dim} \widetilde{M}=\operatorname{dim} I_{0} / K \leq \operatorname{dim} I_{0} / K_{x} \leq \operatorname{dim} \widetilde{M}
$$

where all the inequalities are actually equalities. This means the isometry group $I_{0}$ is acting transitively on $\widetilde{M}$, so that $(\widetilde{M}, \widetilde{g})$ is a symmetric space and the quotient $(M, g)$ is locally symmetric.

Case $2 \Gamma_{0}^{\prime}=1$ The group $I_{0}$ is compact, since it contains $\Gamma_{0}^{\prime}$ as a lattice. On the other hand, we have shown that $I_{0}$ has no compact factors, so $I_{0}=1$, ie, the isometry group $I$ is discrete. Since $\Gamma$ is a lattice in $I$, we conclude that it is a finite index subgroup of $I$. Thus, there is a further finite index subgroup $\Gamma^{\prime \prime}<\Gamma$ that is normalized by $I$. Conjugation by $I$ gives a group homomorphism:

$$
\rho: I / \Gamma^{\prime \prime} \rightarrow \operatorname{Out}\left(\Gamma^{\prime \prime}\right)
$$

An element in the kernel of this homomorphism is a homotopically trivial isometry of $\left(\widetilde{M} / \Gamma^{\prime \prime}, \widetilde{g}\right)$. By Theorem 1, there are no such homotopically trivial isometries, so the map $\rho$ is injective. By Margulis-Mostow-Prasad rigidity, the image $\rho\left(I / \Gamma^{\prime \prime}\right)$ can be represented by isometries of the locally symmetric metric $h_{\text {sym }}$. Thus, the group $L$ of lifts

$$
1 \rightarrow \Gamma^{\prime \prime} \rightarrow L \rightarrow \rho\left(I / \Gamma^{\prime \prime}\right) \rightarrow 1
$$

can be represented by a discrete group of isometries of the symmetric metric. This extension is determined by $\rho$ since $\Gamma^{\prime \prime}$ has trivial center (Proposition 9) so the group of lifts $L$ is isomorphic to $I$. We have shown that $I$ is a group of isometries of the symmetric metric. Let $\Gamma$ act on the universal cover $\widetilde{M}$ by isometries via:

$$
\Gamma<I \cong L<\operatorname{Isom}\left(\widetilde{M}, \tilde{h}_{\text {sym }}\right)
$$


By Margulis-Mostow-Prasad rigidity, the quotient $\left(\widetilde{M} / \Gamma, \tilde{h}_{\mathrm{sym}}\right)$ is isometric to $\left(M, h_{\text {sym }}\right)$. Consequently,

$$
|I / \Gamma|=\frac{\operatorname{vol}\left(\widetilde{M} / \Gamma, \widetilde{h}_{\mathrm{sym}}\right)}{\operatorname{vol}\left(\widetilde{M} / I, \widetilde{h}_{\mathrm{sym}}\right)} \leq \frac{\operatorname{vol}\left(M, h_{\mathrm{sym}}\right)}{\varepsilon\left(h_{\mathrm{sym}}\right)}
$$

where $\varepsilon\left(h_{\text {sym }}\right)$ is the volume of the smallest locally symmetric orbifold covered by $\left(M, h_{\text {sym }}\right)$. This completes the proof of Theorem 2 .

Remark 6 If $\left(\widetilde{M}, \widetilde{h}_{\text {sym }}\right)$ is a symmetric space with no compact or Euclidean factors, then the volume of the smallest locally symmetric orbifold isometrically covered by $\left(\widetilde{M}, \tilde{h}_{\text {sym }}\right)$ is bounded below by a positive constant $\varepsilon\left(\tilde{h}_{\text {sym }}\right)$ that depends only on the symmetric space. This is a theorem of Kazhdan and Margulis (cf XI.11.9 in [17]). By contrast, the real line covers circles of arbitrarily small volume. Similarly, any symmetric space with a compact or Euclidean factor will support compact quotients of arbitrarily small volume.

\section{References}

[1] G Avramidi, Smith theory, $L^{2}$ cohomology, isometries of locally symmetric manifolds and moduli spaces of curves arXiv:math.GT/1106.1704

[2] M Bestvina, M Feighn, Proper actions of lattices on contractible manifolds, Invent. Math. 150 (2002) 237-256 MR1933585

[3] A Borel, J-P Serre, Corners and arithmetic groups, Comment. Math. Helv. 48 (1973) 436-491 MR0387495

[4] G E Bredon, Sheaf theory, Graduate Texts in Mathematics 170, Springer, New York (1997) MR1481706

[5] P E Conner, F Raymond, Manifolds with few periodic homeomorphisms, from: "Proceedings of the second conference on compact transformation groups, II", (H T Ku, L N Mann, J L Sicks, J C Su, editors), Lecture Notes in Math. 299, Springer, Berlin (1972) 1-75 MR0358835

[6] P B Eberlein, Geometry of nonpositively curved manifolds, Chicago Lectures in Mathematics, University of Chicago Press (1996) MR1441541

[7] B Farb, S Weinberger, Isometries, rigidity and universal covers, Ann. of Math. 168 (2008) 915-940 MR2456886

[8] B Farb, S Weinberger, The intrinsic asymmetry and inhomogeneity of Teichmüller space, Duke Math. J. 155 (2010) 91-103 MR2730373

[9] C Löh, R Sauer, Degree theorems and Lipschitz simplicial volume for nonpositively curved manifolds of finite volume, J. Topol. 2 (2009) 193-225 MR2499443 
[10] S Mac Lane, Homology, Classics in Mathematics, Springer, Berlin (1995) MR1344215

[11] K Melnick, Compact Lorentz manifolds with local symmetry, J. Differential Geom. 81 (2009) 355-390 MR2472177

[12] D Morris, Introduction to arithmetic groups arXiv:math.GT/0106063

[13] S B Myers, N E Steenrod, The group of isometries of a Riemannian manifold, Ann. of Math. 40 (1939) 400-416 MR1503467

[14] T T Nguyen Phan, Minimal orbifolds and (a)symmetry of piecewise locally symmetric manifolds arXiv:math.GT/1110.0438

[15] A Pettet, J Souto, Periodic maximal flats are not peripheral arXiv: math.GT/0909. 2899

[16] G Prasad, Discrete subgroups isomorphic to lattices in Lie groups, Amer. J. Math. 98 (1976) 853-863 MR0480866

[17] MS Raghunathan, Discrete subgroups of Lie groups, Math. Student (2007) 59-70 MR2527560

[18] W Thurston, The geometry and topology of three-manifolds, unpublished notes (1978)

[19] R J Zimmer, Ergodic theory and semisimple groups, Monographs in Mathematics 81, Birkhäuser, Basel (1984) MR776417

Department of Mathematics, University of Chicago

5734 S. University Avenue, Chicago, IL 60637, USA

gavramid@math. uchicago.edu

math.uchicago.edu/ gavramid

Proposed: Steve Ferry

Received: 1 August 2011

Seconded: Walter Neumann, Jean-Pierre Otal

Revised: 10 October 2012 